\title{
The NAD(P)H:quinone oxidoreductase I C609T polymorphism modifies the risk of Barrett esophagus and esophageal adenocarcinoma
}

Erica di Martino, $P h D^{1}$, Laura J. Hardie, PhD ${ }^{1}$, Christopher P. Wild, $P h D^{1}$, Yun Y. Gong, PhD ${ }^{1}$, Joanna R. Olliver, $P h D^{1}$, Martin D. Gough, MB ChB MRCS (Ed. $)^{2}$, and Nigel C. Bird, MEd, PhD ${ }^{2}$

\begin{abstract}
Purpose: The role of genetic susceptibility to esophageal adenocarcinoma and its precursor lesion Barrett esophagus has not been fully elucidated. This study investigated the effect of polymorphisms in the manganese superoxide dismutase $(M n S O D)$ and $\mathrm{NAD}(\mathrm{P}) \mathrm{H}$ :quinone oxidoreductase 1 (NQO1) genes in modulating the risk of developing Barrett esophagus or esophageal adenocarcinoma. Methods: A total of 584 patients (146 esophagitis, 200 Barrett esophagus, 144 esophageal adenocarcinoma, and 94 controls) were genotyped for the MnSOD C14T and NQO1 C609T polymorphisms using polymerase chain reaction and restriction fragment length polymorphism analysis. Results: The NQO1 TT genotype was less common in Barrett esophagus (2.0\%) and esophageal adenocarcinoma (1.4\%) patients, compared with both esophagitis patients (7.6\%) and controls (5.4\%). After adjustment for sex, age, body mass index, reflux symptoms, and smoking status, patients with the homozygous TT genotype had a 4.5 -fold decreased risk of developing Barrett esophagus (odds ratio $=0.22,95 \%$ confidence interval $=0.07-0.76, P=0.01$ ) and a 6.2 -fold decreased risk of esophageal adenocarcinoma (odds ratio $=0.16$, $95 \%$ confidence intervals $=0.03-0.94, P=0.04$ ) compared with individuals with the TC and CC genotypes. No significant differences between groups were observed for the MnSOD polymorphism $(P=0.289)$. Conclusions: Overall, the results of this study suggest that the NQO1 TT genotype may offer protection from reflux complications such as Barrett esophagus and esophageal adenocarcinoma. Genet Med 2007:9(6):341-347.
\end{abstract}

Key Words: adenocarcinoma, Barrett epithelium, esophagus, manganese superoxide dismutase, NAD(P)H:quinone oxidoreductase 1

Most esophageal adenocarcinomas (EACs) arise as the last stage of a multistep pathway beginning with a metaplastic change in a segment of esophageal mucosa, a condition known as Barrett esophagus (BE). ${ }^{1}$ In this premalignant stage, the pluristratified esophageal epithelium acquires columnar, intestine-like characteristics, including the presence of goblet cells. ${ }^{2}$ Over the past 30 years, a number of epidemiological studies have established a causative link between gastroesophageal reflux disease (GERD) and the development of BE and EAC, with the risk increased in individuals with the greatest severity, duration, and frequency of reflux. ${ }^{3,4}$ Despite the clear link between reflux and the risk of BE and EAC, only a minority of GERD patients develop reflux-related complications, and as

\footnotetext{
From the ${ }^{1}$ Molecular Epidemiology Unit, Centre for Epidemiology and Biostatistics, Leeds Institute for Genetics, Health and Therapeutics, University of Leeds, Leeds, United Kingdom; ${ }^{2}$ Academic Surgical Unit, University of Sheffield, Sheffield, United Kingdom.

Laura Hardie, PhD, Molecular Epidemiology Unit, LIGHT Laboratories, Clarendon Way, University of Leeds, Leeds LS2 9JT, UK; E-mail: l.j.hardie@leeds.ac.uk

Disclosure: The authors declare no conflict of interest.

Submitted for publication November 30, 2006.

Accepted for publication February 15, 2007.

DOI: 10.1097/GIM.0b013e3180654ccd
}

few as $0.5 \%$ of BE patients progress to cancer each year. ${ }^{5}$ As yet, the reasons for such heterogeneity in response to reflux remain to be clarified.

Accumulating evidence suggests that free radicals may be important mediators of the damaging effects of reflux. In animal models of GERD, for example, acid- and pepsin-induced esophagitis was significantly reduced by supplementation with the free radical scavenger superoxide dismutase (SOD). ${ }^{6}$ Furthermore, SOD supplementation also prevented progression of esophagitis to BE and EAC. ${ }^{7}$ Similarly, a diet rich in antioxidants such as vitamin $\mathrm{C}, \beta$-carotene, and $\alpha$-tocopherol, appears to reduce EAC risk, particularly in people with reflux. ${ }^{8}$ Oxidative damage in GERD patients may arise from the inflammatory process associated with reflux, with superoxide anion and hydrogen peroxide produced by activated leukocytes and macrophages. ${ }^{9}$ Exposure of the esophageal epithelium to these free radicals may explain the increased levels of DNA damage measured in Barrett epithelium compared with normal esophageal tissue. ${ }^{10}$ The consequent accumulation of DNA damage could contribute to the acquisition of genetic alterations and ultimately lead to malignant transformation in some patients. In addition to the inflammatory response induced by reflux, both endogenous and exogenous components 
of refluxate may also induce DNA damage. Short in vitro exposure of esophageal cells to bile acids, for example, is associated with a significant increase in the level of DNA strand breaks. ${ }^{11}$ In addition, there is strong evidence that as a result of the acid environment created by reflux, the esophagus, and specifically the $\mathrm{BE}$ segment, may be the site of the conversion of nitrite to a number of mutagens, such as nitric oxide, nitrous acid and $N$-nitroso compounds. ${ }^{12-14}$

In view of this mutagenic potential of reflux, the heterogeneity in GERD and BE outcome may be related to individual variation in antioxidant, detoxification, and DNA repair pathways, which could affect the risk of acquiring the genetic alterations observed in the progression of BE to malignancy. A number of case-control studies have investigated the association between specific genetic polymorphisms and esophageal squamous cell carcinoma in high-risk Asian populations. ${ }^{15-19}$ To date, however, only a few reports have been published regarding genetic susceptibility to EAC in Western countries; still fewer have addressed the role of genetic susceptibility to $\mathrm{BE}$.

In this study, we examined the role of genetic polymorphisms in the manganese superoxide dismutase $(\mathrm{MnSOD})$ and $\mathrm{NAD}(\mathrm{P}) \mathrm{H}$ :quinone oxidoreductase 1 (NQO1) genes in modulating susceptibility to BE and EAC in a UK population. MnSOD was selected for investigation because this enzyme provides an important antioxidant defense against the superoxide anions produced during inflammatory processes. ${ }^{8,20}$ The $\mathrm{MnSOD}$ C14T polymorphism results in an alanine (C allele) to valine ( $\mathrm{T}$ allele) amino acid change within the signal peptide responsible for the transport of the mature protein to the correct subcellular location ${ }^{21,22}$ and has been associated with susceptibility to a variety of cancers. ${ }^{23-27}$ The phase II detoxification enzyme NQO1 was chosen because it reacts with a number of natural and synthetic compounds including quinones, quinone-epoxides, quinone-imines, naphthoquinones, methylene blue, azo- and nitro-compounds ${ }^{28}$ and could therefore metabolize a wide range of dietary carcinogens contained in reflux. The TT genotype corresponds to a null phenotype, as it results in the production of a mutated protein with reduced activity and increased half-life. ${ }^{29}$ Previous studies have shown a link between this polymorphism and a number of cancers, including gastroesophageal malignancies..$^{30,31}$

The results of our study suggest that the NQO1 C609T polymorphism may modulate the likelihood of patients with gastroesophageal reflux developing $\mathrm{BE}$ and $\mathrm{EAC}$, whereas no association was found between the $M n S O D C 14 \mathrm{~T}$ polymorphism and the risk of these diseases.

\section{MATERIALS AND METHODS}

\section{Patient groups}

After informed consent, a total of 584 patients were recruited from the dedicated dyspepsia and Barrett endoscopy clinics of two tertiary referral centers (Royal Hallamshire Hospital, Sheffield and Leeds General Infirmary, Leeds) in the Yorkshire region of the United Kingdom from 2000 to 2003. Information about age, sex, reflux symptoms, body mass index
(BMI), smoking history, alcohol consumption, and medication was collected at recruitment. DNA was extracted from blood ( $n=470)$ or normal esophageal biopsy samples $(n=$ 114), using a phenol/ethanol method or the TriReagent kit (Sigma-Aldrich Ltd., Gillingham, UK).

Based on endoscopic and histological evidence, patient groups included symptomatic controls $(n=94)$, esophagitis $(n=146)$, Barrett $(n=200)$, and cancer $(n=144)$ cases. All control patients were free of endoscopically visible esophageal disease, including esophagitis, columnar epithelium, BE, and EAC. However, as control individuals had been recruited from a dyspepsia endoscopy list, 44 of these (47\%) reported refluxrelated symptoms, such as heartburn and/or regurgitation. Other indications for endoscopy in the patients designated as controls were dyspepsia, epigastric abdominal pain, dysphagia, diarrhea, weight loss, nausea, and suspected celiac disease. $\mathrm{Pa}$ tients included in the esophagitis group had histologically confirmed reflux-related mucosal inflammation but no evidence of columnar mucosa (including BE), dysplasia, or EAC. The Barrett group comprised patients with any length of histologically confirmed specialized intestinal metaplasia containing goblet cells, of whom 28 (14\%) also had histological evidence of low-grade dysplasia. Finally, the cancer group comprised patients with EAC (90\%) but also 14 individuals with histologically confirmed high-grade dysplasia (HGD). The rationale for including HGD with EAC is that a high proportion of HGD patients are expected to be incident cases of EAC or likely to progress to EAC within a short period. ${ }^{32,33}$ All samples used in this study were collected after obtaining informed consent from patients. Ethical approval for the study was granted by the South Sheffield Research Ethics Committee and the United Leeds Teaching Hospitals Trust.

\section{Polymerase chain reaction (PCR)}

Genotyping for the NQO1 C609T and MnSOD C14T polymorphisms was performed by PCR and restriction length fragment polymorphism analysis, as previously described, ${ }^{23,30}$ with minor modifications. Briefly, $1 \mu \mathrm{L}$ of DNA was amplified with $1 \times$ PCR buffer (Qiagen Ltd., Crawley, UK), 12.5 pmol of forward and reverse primers (InVitrogen Ltd., Paisley, UK), 0.5 units of HotStarTaq DNA Polymerase (Qiagen Ltd.), and $200 \mu \mathrm{M}$ deoxyribonucleoside triphosphate (InVitrogen Ltd.), in a total volume of $20 \mu \mathrm{L}$, using a GeneAmp PCR System 2700 thermocycler (Applied Biosystems, Warrington, UK). Primer sequences were $5^{\prime}$ aagcccagaccaacttct $3^{\prime}$ and $5^{\prime}$ atttgaattcgggcgtctgctg $3^{\prime}$ for NQO1 and 5' accagcaggcagctggcgccgg3' and 5' gcgttgatgtgaggttccag $3^{\prime}$ for $\mathrm{MnSOD}$. Reaction conditions were 15 minutes at $95^{\circ} \mathrm{C}$ to activate the HotStarTaq, followed by 38 cycles of 30 seconds at $95^{\circ} \mathrm{C}, 30$ seconds at $60^{\circ} \mathrm{C}$, and 30 seconds at $72^{\circ} \mathrm{C}$, and 7 minutes of final elongation at $72^{\circ} \mathrm{C}$. Nontemplate controls, in which DNA was omitted, were included in each amplification batch. The success of the PCR reaction and the lack of cross-contamination in the negative controls were checked by $1.5 \%$ agarose gel electrophoresis. Expected sizes of the PCR products were $174 \mathrm{bp}$ for NQO1 and $107 \mathrm{bp}$ for $\mathrm{MnSOD}$. 


\section{Restriction fragment length polymorphism analysis}

Ten microliters of PCR product was digested overnight at $37^{\circ} \mathrm{C}$ with 5 units of HinfI (NQO1) or NgoM IV (MnSOD) (New England Biolabs Ltd., Hitchin, UK) in a total volume of $20 \mu \mathrm{L}$. Genotypes were visualized by $3 \%$ (NQO1) or $4 \%$ $(\mathrm{MnSOD})$ agarose gel electrophoresis of the PCR product in $0.5 \mathrm{x}$ Tris borate ethylene diamine tetracetic acid buffer containing $0.5 \mu \mathrm{g} / \mathrm{mL}$ ethidium bromide (Sigma-Aldrich Ltd., Gillingham, UK). For the NQO1 polymorphism, the C allele was visualized as a 174 bp band, whereas the T allele was digested into two fragments of 122 and $52 \mathrm{bp}$ (Fig. 1). The T allele of the MnSOD polymorphism was identified by an uncut 107 bp product, whereas the $\mathrm{C}$ allele produced two fragments of 89 and 18 bp (Fig. 2). After confirmation of the correct genotype by sequencing (GRI Genomics, Rayne, UK), one sample for each of the three genotypes was included in all amplification and digestion batches as quality controls. Genotyping was repeated for $10 \%$ of the samples and demonstrated consistent results in all instances.

\section{Statistical analysis}

Differences in categorical variables (male/female gender, ever/ never smoker) between study groups were assessed using the Pearson $\chi^{2}$ test. The Kruskal-Wallis test was applied for the comparison of continuous variables (age, BMI, and alcohol intake) because these variables did not fit with a normal distribution, as indicated by the Kolmogorov-Smirnov test. Odds ratios (ORs) and 95\% confidence intervals (CIs) associated with specific genotypes were calculated by multiple logistic regression, with and without adjustment for sex, age, BMI, reflux, and smoking status. Statistical analysis was performed using SPSS 12.0 (SPSS Inc., Chicago, IL) and STATA 7 (STATA Corporation, College Station, TX) software. In addition, genotype frequencies were tested for
Hardy-Weinberg equilibrium using $\chi^{2}$ goodness-of-fit test (Graphpad Instat, 2.05). $P \leq 0.05$ was considered as significant.

\section{RESULTS}

\section{Population characteristics}

Information regarding patient gender, age, BMI, smoking status, and alcohol consumption is reported in Table 1. There were no statistical differences in gender composition between the esophagitis, Barrett, and cancer groups $(P=0.281)$, which as expected included a majority of male subjects. However, the number of males in the symptomatic controls was lower than in the other groups $(P<0.001)$. Significant differences in age were observed between patient groups $(P<0.001)$, with cancer patients tending to be older than Barrett patients, who in turn were older than symptomatic controls and esophagitis patients. No association was found between average BMI $(P=$ $0.144)$, smoking status $(P=0.068)$, or alcohol intake $(P=$ 0.088 ) and risk of $\mathrm{BE}$ and/or EAC.

\section{Genotype frequencies and ORs}

Overall, of the 584 samples included in the study, the number successfully genotyped was 578 for NQO1 and 577 for MnSOD (99\%). Genotype frequencies in all patient groups were in accordance with Hardy-Weinberg equilibrium $(P>0.05)$ except for the NQO1 polymorphism in esophagitis patients $(P=0.005)$. No significant differences in the genotype frequencies for the $\mathrm{Mn}$ SOD polymorphism were observed between groups $(P=0.289)$ (Table 2). In contrast, frequencies of the NQO1 C609T $(P<$ 0.037 ) polymorphism differed across patient groups. The TT NQO1 genotype, was significantly less common in $\mathrm{BE}(2.0 \%, P=$ $0.024)$ and $\mathrm{EAC}(1.4 \%, P=0.030)$ patients compared with individuals with esophagitis (7.6\%). Based on the comparison of ge-

\section{$\begin{array}{llllllllllllllll}1 & 2 & 3 & 4 & 5 & 6 & 7 & 8 & 9 & 10 & \mathrm{~A} & \mathrm{~B} & \mathrm{C} & \mathrm{N} & \mathrm{M}\end{array}$}

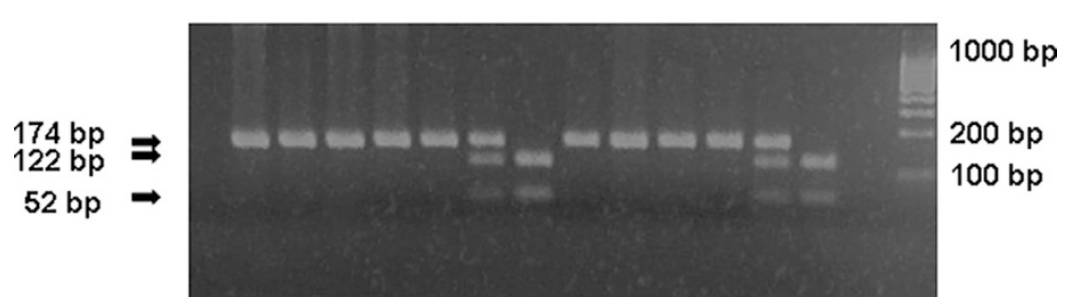

Fig. 1. NQO1 C609T genotypes visualized by $3 \%$ agarose gel electrophoresis. The $\mathrm{C}$ allele appears as a $174 \mathrm{bp}$ band, whereas the $\mathrm{T}$ allele is indicated by two fragments of 122 and $52 \mathrm{bp}$. A, CC control; B, CT control; C, TT control; N, nontemplate control; M, 100-bp ladder. Numbers across the top indicate digested PCR product for separate individuals.

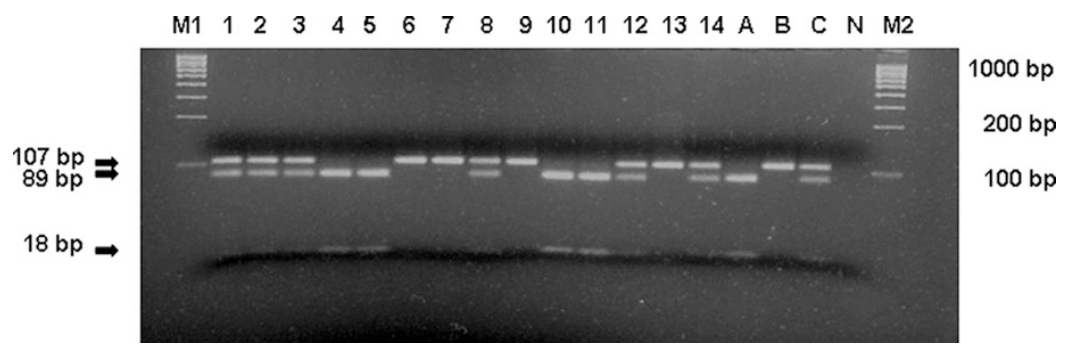

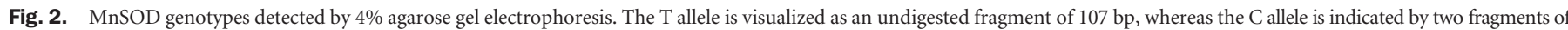

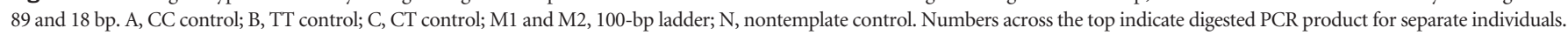


Table 1

Information on gender, age, BMI, smoking, and drinking habits for patients in the four study groups

\begin{tabular}{|c|c|c|c|c|c|}
\hline & $\begin{array}{l}\text { Symptomatic } \\
\text { controls }\end{array}$ & Esophagitis & $\mathrm{BE}$ & EAC & $P$ \\
\hline Total no. & 94 & 146 & 200 & 144 & \\
\hline Male patients, no. (\%) & $42(44.7)$ & $106(72.6)$ & $148(74.0)$ & $117(81.2)$ & $<0.001^{a}$ \\
\hline Average age, y (range) & $56(29-88)$ & $58(22-88)$ & $65(25-97)$ & $72(41-94)$ & $<0.001^{b}$ \\
\hline Average BMI (range) & $28.9(16.8-55.3)$ & $26.4(14.0-41.2)$ & $25.7(17.0-37.6)$ & $26.4(15.4-51.5)$ & 0.144 \\
\hline Ever smokers, no. (\%) & $50(53.2)$ & $87(59.5)$ & $127(63.5)$ & $89(61.8)$ & 0.068 \\
\hline Average units alcohol/wk (range) & $9.0(0-50)$ & $13.8(0-100)$ & $11.7(0-100)$ & $9.7(0-90)$ & 0.088 \\
\hline Patients $>21$ units/wk, no. (\%) & $15(15.9)$ & $25(17.1)$ & $28(14.0)$ & $18(12.5)$ & 0.888 \\
\hline
\end{tabular}

BE, Barrett esophagus; EAC, esophageal adenocarcinoma; BMI, body mass index.

${ }^{a} P<0.001$ between control patients and the other groups; $P=0.281$ between esophagitis, BE, and EAC.

${ }^{b} P=0.203$ between control and esophagitis patients; $P<0.001$ between all other groups.

Table 2

Genotype frequencies for the MnSOD C14T and NQO1 C609T polymorphisms in esophagitis, BE, and EAC cases and in endoscopically normal control individuals

\begin{tabular}{lcccc}
\hline & Symptomatic controls & Esophagitis & BE & $P^{a}$ \\
\hline MnSOD C14T & & & & EAC \\
CC & $36.6 \%(34 / 93)$ & $23.6 \%(34 / 144)$ & $26.5 \%(53 / 200)$ & $25.0 \%(35 / 140)$ \\
CT & $41.9 \%(39 / 93)$ & $46.5 \%(67 / 144)$ & $47.0 \%(94 / 200)$ & $52.1 \%(73 / 140)$ \\
TT & $21.5 \%(20 / 93)$ & $29.9 \%(43 / 144)$ & $26.5 \%(53 / 200)$ & $22.9 \%(32 / 140)$ \\
NQO1 C609T & & & $67.0 \%(134 / 200)$ & $68.1 \%(96 / 141)$ \\
CC & $59.1 \%(55 / 93)$ & $68.1 \%(98 / 144)$ & $31.0 \%(62 / 200)$ & $30.5 \%(43 / 141)$ \\
CT & $35.5 \%(33 / 93)$ & $24.3 \%(35 / 144)$ & $2.0 \%(4 / 200)^{b}$ & $1.4 \%(2 / 141)^{c}$ \\
TT & $5.4 \%(5 / 93)$ & $7.6 \%(11 / 144)^{b, c}$ & & \\
\hline
\end{tabular}

BE, Barrett esophagus; EAC, esophageal adenocarcinoma.

${ }^{a} P$ values indicate significance of differences in genotype frequencies across all groups.

${ }^{b}$ Esophagitis vs. BE $(P=0.024)$.

'Esophagitis vs. EAC $(P=0.030)$

Table 3

Odds ratios and $P$ values associated with the NQO1 C609T genotypes (TT/CC+CT)

\begin{tabular}{|c|c|c|c|c|}
\hline & \multicolumn{2}{|c|}{ OR (95\% CI) } & \multicolumn{2}{|c|}{$P$} \\
\hline & Unadjusted & Adjusted $^{a}$ & Unadjusted & Adjusted $^{a}$ \\
\hline Esophagitis vs. symptomatic controls & $1.45(0.49-4.33)$ & $0.80(0.16-4.06)$ & 0.500 & 0.790 \\
\hline BE vs. symptomatic controls & $0.36(0.09-1.36)$ & $0.34(0.05-2.25)$ & 0.132 & 0.364 \\
\hline BE vs. esophagitis & $0.24(0.08-0.79)$ & $0.22(0.07-0.76)$ & 0.018 & 0.016 \\
\hline EAC vs. symptomatic controls & $0.25(0.05-1.33)$ & $0.12(0.01-1.58)$ & 0.105 & 0.107 \\
\hline EAC vs. esophagitis & $0.17(0.04-0.80)$ & $0.16(0.03-0.94)$ & 0.025 & 0.043 \\
\hline EAC vs. BE & $0.71(0.13-3.92)$ & $0.80(0.13-4.92)$ & 0.693 & 0.806 \\
\hline
\end{tabular}

OR, odds ratio; CI, confidence interval; BE, Barrett esophagus; EAC, esophageal adenocarcinoma.

${ }^{a}$ Adjusted for age, sex, reflux, body mass index, and smoking status.

notype frequencies between the esophagitis group and the $\mathrm{BE}$ and EAC groups, a significantly reduced risk of $\mathrm{BE}(\mathrm{OR}=0.24,95 \%$ $\mathrm{CI}=0.08-0.79, P=0.018)$ and $\mathrm{EAC}(\mathrm{OR}=0.17,95 \% \mathrm{CI}=$ $0.04-0.80, P=0.025)$ was observed in esophagitis patients with the TT NQO1 genotype (Table 3). These results remained essentially unchanged after adjustment for age, sex, BMI, reflux symp- toms, and smoking status, with the TT NQO1 genotype associated to a 4.5 -fold decreased risk of developing $\mathrm{BE}(\mathrm{OR}=0.22,95 \%$ $\mathrm{CI}=0.07-0.76, P=0.01)$ and a 6.2 -fold reduced risk of EAC $(\mathrm{OR}=0.16,95 \% \mathrm{CI}=0.03-0.94, P=0.04)$ (Table 3). A similar trend was observed when genotype frequencies were compared between $\mathrm{BE}$ or EAC patients and symptomatic controls, with a 
protective effect of the TT genotype against $\mathrm{BE}(\mathrm{OR}=0.34,95 \%$ $\mathrm{CI}=0.05-2.25)$ and $\mathrm{EAC}(\mathrm{OR}=0.12,95 \% \mathrm{CI}=0.01-1.58)$, but these differences were not statistically significant $(P=0.364$ and $P=0.107$, respectively) (Table 3 ). The frequency of the TT NQO1 genotype in the control and esophagitis groups were similar $(P=$ 0.167 ) (Table 2), and the TT genotype was not associated with a significant risk of esophagitis $(\mathrm{OR}=1.45,95 \% \mathrm{CI}=0.49-4.33$, $P>0.500)$ (Table 3).

\section{DISCUSSION}

Reflux symptoms are common in Western populations, ${ }^{34,35}$ but when such patients are referred for endoscopy, some have no visible pathology, some have developed esophagitis, and others have developed the precancerous lesion of $\mathrm{BE},{ }^{36}$ which is associated with an increased risk of progression to EAC. In an attempt to improve EAC prognosis with an early diagnosis, the American College of Gastroenterology recommends the enrollment of all $\mathrm{BE}$ patients in endoscopic surveillance programs. ${ }^{37}$ The development of genetic markers to identify the subset of $\mathrm{BE}$ patients more likely to progress to cancer, however, would allow surveillance to be targeted specifically at the individuals at higher risk. The aim of this study was therefore to assess whether genetic polymorphisms in genes related to free radical defense and xenobiotic detoxification could contribute to the heterogeneity in the development of esophageal pathology and could possibly be used as markers of higher risk of progression.

When we examined the effect of the C609T polymorphism in the gene encoding for the detoxification enzyme NQO1, we found no difference in genotype frequencies between control and $\mathrm{BE}$ or EAC patients. In contrast, individuals with esophagitis demonstrated a significantly higher frequency of the TT genotype compared with patients with intestinal metaplasia or cancer. This finding could suggest a protective effect of the $T$ allele against the development of more severe reflux complications, namely, BE and EAC. The mechanism by which the rare $\mathrm{T}$ allele, resulting in a mutated protein with reduced activity, ${ }^{29}$ may confer protection against $\mathrm{BE}$ and $\mathrm{EAC}$ could reside in the ability of NQO1 to activate promutagens contained in reflux. Therefore, one explanation for the lack of significant differences in genotype frequencies across the control and the $\mathrm{BE}$ and EAC groups could relate to the fact that the protective effect of the TT genotype may only be apparent when both cases and controls are subject to the relevant environmental exposure, i.e., longstanding reflux. In our study, only half of the control individuals reported reflux symptoms. In contrast, esophagitis patients reported long-standing reflux symptoms, consistent with $\mathrm{BE}$ and $\mathrm{EAC}$ groups, and could therefore be argued to represent a more suitable control group.

The results of our study are consistent with those of previous investigations showing a similar protective effect of the null $\mathrm{T}$ allele against lung cancer ${ }^{38-41}$ possibly due to the reduced activating effects of NQO1 toward carcinogens contained in tobacco smoke..$^{41}$ Nevertheless, other studies suggest that the decrease in NQO1 activity associated with the $\mathrm{T}$ allele may predispose to gastric, ${ }^{30}$ colon, ${ }^{42}$ bladder, ${ }^{43}$ cervical, ${ }^{44}$ and breast ${ }^{45}$ cancer. The apparent contrasting role of this polymorphism in different malignancies may reflect the complex effects of NQO1 on carcinogen metabolism and its ability to exert both antioxidant and pro-oxidant activities. ${ }^{29,46} \mathrm{NQO} 1$ detoxifies toxic quinones, such as some derivatives of benzene, by catalyzing their reduction into the more stable and readily excreted hydroquinones. The metabolism of other substances, however, may lead to intermediates that can generate free radicals or alkylating species. Some nitroso compounds contained in food and some toxic substances found in diesel exhaust, for example, are activated by NQO1. ${ }^{29,46}$

Regarding the role of NQO1 in esophageal carcinogenesis, in one investigation, the TT genotype was associated with an increased risk of squamous cell carcinoma. ${ }^{31}$ The contrast between the protective effect of high NQO1 activity in esophageal squamous cell carcinoma and our findings of a predisposing role toward EAC could reflect the different etiology of these two histologically distinct types of esophageal cancer. NQO1 may, for instance, activate specific carcinogens involved exclusively in EAC development, such as exogenous and endogenous compounds contained in reflux. Nitroso compounds could be a plausible target for such activation, in view of their emerging role in the development of BE and EAC. ${ }^{12-14}$

In contrast with our results, a predisposing effect of the NQO1 T allele toward EAC was previously suggested in a small study comprising $61 \mathrm{EAC}$ cases, ${ }^{30}$ although a subsequent larger investigation from the same group did not confirm these earlier findings. ${ }^{47}$ The lack of association in the study by von Rahden and colleagues ${ }^{47}$ may be due to the fact that in their investigation, EAC cases were compared with healthy volunteers without reflux. As discussed above, the protective effect of the TT genotype may be apparent only in the presence of procarcinogen contained in reflux. However, in view of these inconsistencies, the role of the NQO1 polymorphism in esophageal carcinogenesis needs to be further clarified in subsequent investigations. In particular, it would be interesting to confirm whether this polymorphism influences the likelihood of progression to $\mathrm{BE}$ and $\mathrm{EAC}$ in the context of a large prospective study enrolling patients with chronic reflux symptoms.

In our study, we also investigated a polymorphism in the gene encoding for the free radical scavenger MnSOD. This enzyme plays a crucial role in defense against free radicals by catalyzing the dismutation of superoxide radicals to hydrogen peroxide, which is then converted to water by catalases and peroxidases. ${ }^{20} \mathrm{~A}$ recent report has shown that MnSOD levels in the esophagus are altered during EAC development, ${ }^{48}$ confirming a possible important protective role of this enzyme against esophageal carcinogenesis. The $\mathrm{C}$ to $\mathrm{T}$ polymorphism examined here results in the replacement of the alanine in position 14 of the MnSOD protein with valine, altering the efficiency of the transport of the mature protein to the correct subcellular localization..$^{21,22}$ As a result, this polymorphism has been shown to modulate predisposition to a number of malignancies, including breast, ${ }^{23,24}$ ovarian, ${ }^{25}$ bladder, ${ }^{26}$ and lung ${ }^{27}$ cancer. Although animal experiments have shown that SOD administration can reduce reflux-mediated esophageal damage, ${ }^{6,7}$ our results suggest that the 
genotype at the $M n S O D C 14 \mathrm{~T}$ locus does not have a major effect on the risk of developing reflux-related complications such as $\mathrm{BE}$ or EAC. However, we cannot exclude a small effect that could be detected in larger studies.

Both esophagitis and BE are common findings in patients with reflux symptoms and referred for endoscopy (37\% and 23\%, respectively). ${ }^{36}$ As both patients and controls in this investigation were recruited from dyspepsia and Barrett endoscopy clinics, a major strength of our study is that all patients had undergone endoscopic examination to allow inclusion into the appropriate study group. As a result, however, our study is limited by the relatively small number of patients recruited, implying that the role of this polymorphism in esophageal carcinogenesis needs to be further clarified in the context of a larger investigation. Furthermore, although our results suggest that the NQO1 TT genotype may protect from progression to EAC, its development as a genetic marker for the identification of patients at lower risk of progression appears unfeasible due to the low frequency of this genotype in disease-free individuals (7.6\%-5.4\%).

In conclusion, our results indicate that the NQO1 C609T polymorphisms may have a role in predisposing GERD patients to reflux complications and that patients with the $T$ allele may have a lower risk of progression to BE and EAC. Larger investigations are required to confirm these findings and to explore the interaction between this genetic polymorphism and specific exposures or with polymorphisms in related genes.

\section{ACKNOWLEDGMENTS}

This research was supported by a PhD studentship to Dr. di Martino from the University of Leeds. Drs. Hardie and Wild are supported by Yorkshire Cancer Research. The authors express their gratitude to the patients who took part in this study and to the clinical staff of the Royal Hallamshire Hospital, Sheffield and Leeds General Infirmary.

\section{References}

1. Wild CP, Hardie LJ. Reflux, Barrett's oesophagus and adenocarcinoma: burning questions. Nat Rev Cancer 2003;3:676-684.

2. Chandrasoma P. Pathological basis of gastroesophageal reflux disease. World J Surg 2003;27:986-993.

3. Lagergren J, Bergstrom R, Lindgren A, Nyren O. Symptomatic gastroesophageal reflux as a risk factor for esophageal adenocarcinoma. N Engl J Med 1999;340:825-831

4. Farrow DC, Vaughan TL, Sweeney C, Gammon MD, et al. Gastroesophageal reflux disease, use of $\mathrm{H} 2$ receptor antagonists, and risk of esophageal and gastric cancer. Cancer Causes Control 2000;11:231-238.

5. Shaheen NJ, Crosby MA, Bozymski EM, Sandler RS. Is there publication bias in the reporting of cancer risk in Barrett's esophagus? Gastroenterology 2000;119:333-338.

6. Naya MJ, Pereboom D, Ortego J, Alda JO, et al. Superoxide anions produced by inflammatory cells play an important part in the pathogenesis of acid and pepsin induced oesophagitis in rabbits. Gut 1997;40:175-181.

7. Piazuelo E, Cebrian C, Escartin A, Jimenez P, et al. Superoxide dismutase prevents development of adenocarcinoma in a rat model of Barrett's esophagus. World J Gastroenterol 2005;11:7436-7443.

8. Terry P, Lagergren J, Ye W, Nyren O, et al. Antioxidants and cancers of the esophagus and gastric cardia. Int J Cancer 2000;87:750-754.

9. Halliwell B, Clement MV, Long LH. Hydrogen peroxide in the human body. FASEB Lett 2000;486:10-13.

10. Olliver JR, Hardie LJ, Dexter S, Chalmers D, et al. DNA damage levels are raised in Barrett's oesophageal mucosa relative to the squamous epithelium of the oesophagus. Biomarkers 2003;8:509-521

11. Jolly AJ, Wild CP, Hardie LJ. Acid and bile salts induce DNA damage in human oesophageal cell lines. Mutagenesis 2004;19:319-324.
12. Casselbrant A, Pettersson A, Ruth M, Bove M, et al. Sources of intra-oesophageal nitric oxide production following intraluminal acid exposure. Scand J Gastroenterol 2002;37:631-637.

13. Iijima K, Henry E, Moriya A, Wirz A, et al. Dietary nitrate generates potentially mutagenic concentrations of nitric oxide at the gastroesophageal junction. Gastroenterology 2002;122:1248-1257.

14. Suzuki H, Iijima K, Scobie G, Fyfe V, et al. Nitrate and nitrosative chemistry within Barrett's oesophagus during acid reflux. Gut 2005;54:1527-1535.

15. Gao CM, Takezaki T, Wu JZ, Li ZY, et al. Glutathione-S-transferases M1 (GSTM1) and GSTT1 genotype, smoking, consumption of alcohol and tea and risk of esophageal and stomach cancers: a case-control study of a high-incidence area in Jiangsu Province, China. Cancer Lett 2002;188:95-102.

16. Yokoyama A, Kato H, Yokoyama T, Tsujinaka T, et al. Genetic polymorphisms of alcohol and aldehyde dehydrogenases and glutathione S-transferase M1 and drinking, smoking, and diet in Japanese men with esophageal squamous cell carcinoma. Carcinogenesis 2002;23:1851-1859.

17. Xing D, Qi J, Miao X, Lu W, et al. Polymorphisms of DNA repair genes XRCC1 and $\mathrm{XPD}$ and their associations with risk of esophageal squamous cell carcinoma in a Chinese population. Int J Cancer 2002;100:600-605.

18. Wang AH, Sun CS, Li LS, Huang JY, et al. Relationship of tobacco smoking CYP1A1 GSTM1 gene polymorphism and esophageal cancer in Xi'an. World J Gastroentero 2002;8:49-53.

19. Yu HP, Wang XL, Sun X, Su YH, et al. Polymorphisms in the DNA repair gene XPD and susceptibility to esophageal squamous cell carcinoma. Cancer Genet Cytogene 2004;154:10-15.

20. Mates JM, Sanchez-Jimenez FM. Role of reactive oxygen species in apoptosis: implications for cancer therapy. Int J Biochem Cell Biol 2000;32:157-170.

21. Shimoda-Matsubayashi S, Matsumine H, Kobayashi T, Nakagawa-Hattori Y, et al. Structural dimorphism in the mitochondrial targeting sequence in the human manganese superoxide dismutase gene. A predictive evidence for conformational change to influence mitochondrial transport and a study of allelic association in Parkinson's disease. Biochem Biophys Res Commun 1996;226:561-565.

22. Rosenblum JS, Gilula NB, Lerner RA. On signal sequence polymorphisms and diseases of distribution. Proc Natl Acad Sci U S A 1996;93:4471-4473.

23. Mitrunen K, Sillanpaa P, Kataja V, Eskelinen M, et al. Association between manganese superoxide dismutase (MnSOD) gene polymorphism and breast cancer risk. Carcinogenesis 2001;22:827-829.

24. Ambrosone CB, Freudenheim JL, Thompson PA, Bowman E, et al. Manganese superoxide dismutase (MnSOD) genetic polymorphisms, dietary antioxidants, and risk of breast cancer. Cancer Res 1999;59:602-606.

25. Olson SH, Carlson MD, Ostrer H, Harlap S, et al. Genetic variants in SOD2, MPO, and NQO1, and risk of ovarian cancer. Gynecol Oncol 2004;93:615-620.

26. Hung RJ, Boffetta P, Brennan P, Malaveille C, et al. Genetic polymorphisms of MPO, COMT, MnSOD, NQO1, interactions with environmental exposures and bladder cancer risk. Carcinogenesis 2004;25:973-978.

27. Wang LI, Miller DP, Sai Y, Liu G, et al. Manganese superoxide dismutase alanineto-valine polymorphism at codon 16 and lung cancer risk. J Natl Cancer Inst 2001; 93:1818-1821.

28. Smith M. Benzene. NQO1, and genetic susceptibility to cancer. Proc Natl Acad Sci US A 1999;96:7624-7626.

29. Ross D, Kepa JK, Winski SL, Beall HD, et al. NAD(P)H:quinone oxidoreductase 1 (NQO1): chemoprotection, bioactivation, gene regulation and genetic polymorphisms. Chem Biol Interact 2000;129:77-97.

30. Sarbia M, Bitzer M, Siegel D, Ross D, et al. Association between NAD (P)H: quinone oxidoreductase 1 (NQ01) inactivating C609T polymorphism and adenocarcinoma of the upper gastrointestinal tract. Int J Cancer 2003;107:381-386.

31. Zhang J, Schulz WA, Li Y, Wang R, et al. Association of NAD $(\mathrm{P}) \mathrm{H}$ : quinone oxidoreductase 1 (NQO1) C609T polymorphism with esophageal squamous cell carcinoma in a German Caucasian and a northern Chinese population. Carcinogenesis 2003;24:905-909.

32. Miros M, Kerlin P, Walker N. Only patients with dysplasia progress to adenocarcinoma in Barrett's oesophagus. Gut 1991;32:1441-1446.

33. Reid BJ, Blount PL, Rubin CE, Levine DS, et al. Flow-cytometric and histologica progression to malignancy in Barrett's esophagus: Prospective endoscopic surveillance of a cohort. Gastroenterology 1992;102:1212-1219.

34. Isolauri J, Laippala P. Prevalence of symptoms suggestive of gastro-oesophagea reflux disease in an adult population. Ann Med 1995;27:67-70.

35. Locke GR III, Talley NJ, Fett SL, Zinsmeister AR, et al. Prevalence and clinica spectrum of gastroesophageal reflux: a population-based study in Olmsted County, Minnesota. Gastroenterology 1997;112:1448-1456.

36. Csendes A, Smok G, Burdiles P, Quesada F, et al. Prevalence of Barrett's esophagus by endoscopy and histologic studies: a prospective evaluation of 306 control subjects and 376 patients with symptoms of gastroesophageal reflux. Dis Esophagus 2000;13: $5-11$. 
37. Sampliner RE. Practice guidelines on the diagnosis, surveillance, and therapy of Barrett's esophagus. The Practice Parameters Committee of the American College of Gastroenterology. Am J Gastroenterol 1998;93:1028-1032.

38. Chen H, Lum A, Seifried A, Wilkens LR, et al. Association of the NAD(P)H:quinone oxidoreductase $609 \mathrm{C}->\mathrm{T}$ polymorphism with a decreased lung cancer risk. Cancer Res 1999;59:3045-3048.

39. Hamajima N, Matsuo K, Iwata $\mathrm{H}$, Shinoda $\mathrm{M}$, et al. $\mathrm{NAD}(\mathrm{P}) \mathrm{H}$ : quinone oxidoreductase 1 (NQO1) C609T polymorphism and the risk of eight cancers for Japanese. Int J Clin Oncol 2002;7:103-108.

40. Lin P, Hsueh YM, Ko JL, Liang YF, et al. Analysis of NQO1, GSTP1, and MnSOD genetic polymorphisms on lung cancer risk in Taiwan. Lung Cancer 2003;40:123-129.

41. Bock CH, Wenzlaff AS, Cote ML, Land SJ, et al. NQO1 T allele associated with decreased risk of later age at diagnosis lung cancer among never smokers: results from a population-based study. Carcinogenesis 2005;26:381-386.

42. Lafuente MJ, Casterad X, Trias M, Ascaso C, et al. NAD(P)H:quinone oxidoreductase-dependent risk for colorectal cancer and its association with the presence of K-ras mutations in tumors. Carcinogenesis 2000;21:1813-1819.
43. Park SJ, Zhao H, Spitz MR, Grossman HB, et al. An association between NQO1 genetic polymorphism and risk of bladder cancer. Mutat Res 2003;536:131137.

44. Niwa Y, Hirose K, Nakanishi T, Nawa A, et al. Association of the NAD(P)H: quinone oxidoreductase C609T polymorphism and the risk of cervical cancer in Japanese subjects. Gynecol Oncol 2005;96:423-429.

45. Menzel HJ, Sarmanova J, Soucek P, Berberich R, et al. Association of NQO1 polymorphism with spontaneous breast cancer in two independent populations. $\mathrm{Br}$ Cancer 2004;90:1989-1994.

46. Cadenas E. Antioxidant and prooxidant function of DT-diaforase in quinone metabolism. Biochem Pharmacol 1995;49:127-140.

47. Von Rahden BHA, Stein HJ, Langer R, von Weyhern CW, et al. C609T polymorphism of the $\mathrm{NAD}(\mathrm{P}) \mathrm{H}$ :quinone oxidoreductase I gene does not significantly affect susceptibility for esophageal adenocarcinoma. Int J Cancer 2005;113:506-508.

48. Hermann B, Li Y, Ray MB, Wo JM, et al. Association of manganese superoxide dismutase expression with progression of carcinogenesis in Barrett esophagus. Arch Surg 2005;140:1204-1209. 